Research Article

\title{
Consecutively Preparing D-Xylose, Organosolv Lignin, and Amorphous Ultrafine Silica from Rice Husk
}

\author{
Hongxi Zhang, ${ }^{1,2}$ Xuefeng Ding, ${ }^{2}$ Zichen Wang, ${ }^{2}$ and Xu Zhao ${ }^{2}$ \\ ${ }^{1}$ Chemistry Department, Changji University, No. 77, North Beijing Road, Changji 831100, China \\ ${ }^{2}$ College of Chemistry, Jilin University, No. 2699, Qianjin Street, Changchun 130012, China \\ Correspondence should be addressed to Xu Zhao; zhaoxu@jlu.edu.cn
}

Received 6 May 2014; Accepted 8 July 2014; Published 22 July 2014

Academic Editor: Takao Yagi

Copyright (c) 2014 Hongxi Zhang et al. This is an open access article distributed under the Creative Commons Attribution License, which permits unrestricted use, distribution, and reproduction in any medium, provided the original work is properly cited.

\begin{abstract}
Rice husk is an abundant agricultural by-product reaching the output of 80 million tons annually in the world. The most common treatment method of rice husk is burning or burying, which caused serious air pollution and resource waste. In order to solve this problem, a new method is proposed to comprehensively utilize the rice husk in this paper. Firstly, the D-xylose was prepared from the semicellulose via dilute acid hydrolysis. Secondly, the lignin was separated via organic solvent pulping from the residue. Finally, the amorphous ultrafine silica was prepared via pyrolysis of the residue produced in the second process. In this way, the three main contents of rice husk (semicellulose, lignin, and silica) are consecutively converted to three fine chemicals, without solid waste produced. The yields of D-xylose and organosolv lignin reach $58.2 \%$ and $58.5 \%$, respectively. The purity and specific surface of amorphous ultrafine silica reach $99.92 \%$ and $225.20 \mathrm{~m}^{2} / \mathrm{g}$.
\end{abstract}

\section{Introduction}

Biomaterials offer the potential of renewability, biodegradation, and a path away from harmful additives. So the biomaterials hold great promise to mitigate many sustainability problems. As one of the biomaterials, rice husk (RH) is the abundant agricultural by-product in all rice producing countries. The annual output of RH of the world and China is around 80 and 40 million tons [1], respectively. Earlier reports had discussed the conversion of rice husk into some products, such as building materials [2], adsorbent [3], and biogas [4]. However, most of them are burnt openly or buried currently in China, which aroused the serious environmental pollution. Therefore, it is very urgent to find new methods to fully utilize the rice husk.

RH is composed of $18 \%$ xylan, $22 \%$ lignin, $38 \%$ cellulose, $20 \%$ amorphous silica, and $2 \%$ other organic components [5]. So the key to comprehensively utilize rice husk is the synchronous utilization of those main contents. The research reported here involves the efficient techniques for consecutively producing $\mathrm{D}$-xylose, organosolv lignin, and amorphous ultrafine silica from the three main contents of rice husk.
D-Xylose is widely used in fields related to the food and pharmaceutical industries for its low calorific value and acceptable organoleptic properties. According to the research of Watanabe et al. [6], the semicellulose in RH is made up of substituted arabinoxylan, so D-xylose can be prepared via dilute acid hydrolysis [7].

Lignin is the abundant renewable aromatic biopolymer [8], which is widely used as adsorbent [9], carbon precursor for pesticide [10], active carbon [11], polymeric additive [12], and so forth. Lignin is separated from biomass via alkaline pulping (Kraft and Soda) process on the commercial scale [13]. However, this method is not suitable for separating the lignin from $\mathrm{RH}$. The amorphous silica in $\mathrm{RH}$ can be easily transformed to the silicate and dissolved during the alkaline pulping process, which causes the loss of silica and difficulty of alkaline recycle. Organic solvent pulping is a good choice to separate lignin from $\mathrm{RH}$, for it has little impact on the amorphous silica of RH. The ethanediol is chosen in this paper as the solvent for the high boiling point and good thermostability.

Ultrafine silica is widely used in electronics, ceramics, plastics, rubber, and photoelectric material industries [14]. 
The content of amorphous silica in $\mathrm{RH}$ is the highest in all Gramineae plants. Preparation of amorphous ultrafine silica from $\mathrm{RH}$ is the most attractive utilization method. Several reports have concluded that rice husks are an excellent source of high-grade amorphous silica [15-17]. We had reported previously that the ultrafine amorphous silica powder is obtained after the hydrolysis of semicellulose and porphyrization of $\mathrm{RH}$ residue [18]. However, the abundant lignin in residue is burned during the pyrolysis process at high temperature $\left(750^{\circ} \mathrm{C}\right)$, the lignin is wasted, and the energy-consuming is high, not to mention the serious aggregation of ultrafine silica due to the high pyrolysis temperature. Therefore, how to effectively utilize the lignin in rice husk, decrease the temperature of pyrolysis, and increase the dispersity of ultrafine silica is discussed in this paper. A new method of comprehensively utilizing the semicellulose, lignin, and silica of rice husk was proposed. In this method, the semicellulose, lignin, and silica in the $\mathrm{RH}$ are consecutively converted to the D-xylose, organosolv lignin, and amorphous ultrafine silica in the way of carbon neutral and without producing solid waste. This method is helpful to resolve the problem of waste and pollution aroused by the rice husk.

\section{Materials and Methods}

2.1. Materials and Reagents. Rice husk is obtained from a rice mill nearby Changchun city, China. RH is washed thoroughly with distilled water to remove adhering soil and dust, then dried at $65^{\circ} \mathrm{C}$ overnight, and porphyrized to 60 meshes before employment.

All chemical reagents, such as ethanediol, sulphuric acid, and calcium oxide, are analytically pure from Sinopharm Chemical Regent Co. Ltd in China.

2.2. Semicellulose Hydrolysis and D-Xylose Preparation. The semicellulose in $\mathrm{RH}$ is hydrolyzed by dilute sulfuric acid under certain conditions. After being filtrated and washed with ethanol, the D-xylose is obtained with a purity of $98.5 \%$. The detailed information is described previously [18].

2.3. Separating Lignin from Residue 1. Residue 1 referred to the residue of rice husk produced in the semicellulose hydrolysis. It is mixed with ethanediol solution at some proportion into a $50 \mathrm{~mL}$ stainless steel autoclave with Teflon inner lining. The autoclave is screwed up and put into an oven heated beforehand to a designed temperature and kept in a given period. After pulping is finished, the autoclave is taken out and naturally cooled to $333 \mathrm{~K}$. The solid RH residue and liquid are rapidly filtrated, and the residue is washed with $333 \mathrm{~K}$ organic solution twice to reduce lignin deposit. The filtrate is gathered in a beaker, and triple volume of ethanediol solution distilled water is supplemented into the filtrate. The automatic precipitation of lignin takes place immediately. For the purpose of complete deposition, electromagnetic stirring is carried out. The suspension is filtrated again to separate solid lignin from spent liquid. The gained lignin is dried overnight at room temperature in the vacuum drying oven.

2.4. Preparation of Amorphous Ultrafine Silica from Residue 2. The two-step method is employed to decrease the temperature and improve the dispersity of ultrafine silica. Residue 2 referred to the $\mathrm{RH}$ produced in the lignin extraction process. After being dried at $65^{\circ} \mathrm{C}$ overnight, residue 2 is weighted and put into a tube furnace, which is heated to a certain temperature beforehand under the flowing of carbon dioxide gas (the first step); residue 2 is hydrolyzed to be black powder (a mixture of silica and carbon). Then the carbon dioxide gas flow is changed to be the oxygen flow under certain temperature (the second step). The black powder completely turns into white silica in 30 minutes.

2.5. Analysis. The size and morphology of silica powder are performed with a transmission electron microscope (JEOL, JEM-1200EX).

The thermal stability of the samples was performed with a thermogravimetric analyzer (Mettler Toledo, TGA/SDTA 851) in air atmospheres. Samples of about $15 \mathrm{mg}$ were heated from room temperature to $900^{\circ} \mathrm{C}$ with the constant heating rate of $10^{\circ} \mathrm{C} / \mathrm{min}$ and the gas flow rate of $20 \mathrm{~mL} / \mathrm{min}$.

The specific surface area and pore size of silica powder are measured by using the Brunauer-Emmett-Teller (BET) method with an automated chemisorption/physisorption surface area and pore size analyzer (Quantachrome AUTOSORB-1C).

The apparent weight-average molecular weight (Mw), the apparent number-average molecular weight $(\mathrm{Mn})$, and the molecular weight distribution (Mw/Mn, DPI) are measured on a Gel Permeation Chromatography (WATERS 1515) instrument. The Styragel HT columns used THF as an eluent $(1.0 \mathrm{~mL} / \mathrm{min})$ at $308 \mathrm{~K}$. The calibration curve is obtained with linear polystyrene as standards.

Lignin is ground and mixed with $\mathrm{KBr}$ to make a pellet $(0.5 \% \mathrm{~m} / \mathrm{m})$ to obtain infrared spectra with Fourier Transform Infrared spectroscopy (Shimadzu FTIR 8400).

Ultraviolet spectroscopy (UV) is carried out on a Shimadzu UV-2550 by using lignin mentioned above. Lignin and barium sulfate are mixed and pressed in the attachment of UV-2550 to get a smooth surface; then the ultraviolet adsorption spectrum of lignin is obtained by integrating sphere method.

The amorphous state of silica powder is performed on an X-ray diffractometer (Shimadzu-6000), using $\mathrm{Cu} \mathrm{K} \alpha(\lambda=$ $1.54056)$ radiation.

To measure the purity of silica powder, inductively coupled plasma (ICP) is performed on an ICP spectrometer (Agilent 7500A).

The content of the semicellulose, cellulose, lignin, and silica in the $\mathrm{RH}$ is measured according to the China National Standards GB/T 2677.9-94, GB/T 2677.10-95, GB/T 747-2003, and GB/T 2677.3-93.

\section{Results and Discussions}

The main contents of rice husk and residues are shown in Table 1. It is seen that the content of lignin, cellulose, and silica increased in residue 1 and residue 2, with the decrement of semicellulose. 
TABLE 1: The main contents of the rice husk, residue 1 , and residue 2.

\begin{tabular}{lccccc}
\hline Content (wt.\%) & Semicellulose & Lignin & Cellulose & Silica & Others \\
\hline Rice husk & 18.20 & 24.52 & 35.86 & 48.85 & 2.57 \\
Residue 1 & 2.42 & 30.23 & 40.61 & 25.48 & 1.26 \\
Residue 2 & - & 4.44 & 56.09 & 38.91 & 0.57 \\
\hline
\end{tabular}

"-" represents the content was undetected.

3.1. Discussion on the Ethanediol Pulping of RH Residue 1. The mechanism of organosolv pulping is commonly considered as follows [19]: the $\alpha$-aryl ether bond and $\beta$-aryl ether bond of lignin are attacked by the organic solution under the conditions of sufficient temperature, pressure, and time. The hydrolysis of $\alpha$-aryl ether bond is the key factor affecting the organosolv pulping, but there are other reactions that occurred at the same time, such as the hydrolysis of $\beta$-aryl ether bond. The lignin matrix is broken and converted to the lignin fragment and then dissolved in the organic solution to form a stable colloidal dispersion. When the water is added in this colloidal dispersion, the concentration of organic solution is decreased; also the dissolvability of lignin fragment is decreased. So the lignin fragment is deposited and separated from the organic solution.

Therefore, the variables affecting the yield of lignin from residue 1 are considered as temperature, time, concentration of ethanediol solution, and the ratio of residue to ethanediol. The method of one factor experiments is carried out for investigating the effects of those variables on the yield of lignin in the pulping process. The yield of lignin in this paper is defined as (actual output of lignin)/(theoretical output of lignin) $\times 100$.

3.2. The Influence of Pulping Temperature on the Yield of Lignin. The influence of pulping temperature on the yield of lignin is investigated, that is, $453 \mathrm{~K}, 463 \mathrm{~K}, 473 \mathrm{~K}, 483 \mathrm{~K}$, and $493 \mathrm{~K}$. Autoclaves were nonisothermally heated to certain temperature and kept for 3 hours, respectively. The concentration of ethanediol was fixed at $80 \%(\mathrm{v} / \mathrm{v})$; the $\mathrm{RH}$ residue 1 and ethanediol solution in fixed ratio $(1: 6, \mathrm{~g}: \mathrm{mL})$ were mixed in stainless steel autoclaves. The lignin samples were obtained after filtration and vacuum-drying at room temperature. The effects of temperature on the yield of lignin are shown in Figure 1(a).

It is seen from Figure 1(a) that the yield of lignin increased rapidly with the increment of temperature from $453 \mathrm{~K}$ to $483 \mathrm{~K}$, but when the temperature exceeded $483 \mathrm{~K}$, the yield of lignin fell off. It can be explained as the hydrolysis of lignin needing enough energy, but when the temperature exceeded $483 \mathrm{~K}$, the side reaction of lignin increased rapidly.

3.3. The Influence of Pulping Time on the Yield of Lignin. The effect of time, that is, $1,2,3,4$, and 5 hours, was investigated. The concentration of ethanediol solution and temperature were fixed at $80 \%$ and $483 \mathrm{~K}$. Residue 1 and ethanediol solution were mixed in fixed ratio of $1: 6(\mathrm{~g}: \mathrm{mL})$ in the stainless steel autoclaves. Those autoclaves are nonisothermally heated to $483 \mathrm{~K}$ and, respectively, kept for a certain time. After filtration and vacuum-drying at room temperature, lignin samples were obtained. The effects of pulping time on the yield of lignin are shown in Figure 1(b).

Figure 1(b) shows a remarkable increment of the lignin's yield with the increment of time before 5 hours. The delignification of biomass in organic solution was modeled as the irreversible and consecutive dissolution of initial, bulk, and residual lignin [20]. As we can see from Figure 1(b), the duration of 2 hours might be the initial dissolution of lignin, 3-4 hours the bulk, and 5 hours the residual dissolution of lignin.

3.4. Influence of Concentration of Ethanediol Solution on the Yield of Lignin. A series of ethanediol solutions with the concentration of $50 \%, 60 \%, 70 \%, 80 \%$, and $90 \%$ (volume fraction) were, respectively, investigated. Then the autoclaves were nonisothermally heated to $483 \mathrm{~K}$ and kept for a further four hours. After being filtrated and vacuum-dried at room temperature, lignin samples were obtained. The influence of concentration on the yield of lignin is shown in Figure 1(c).

It is seen from Figure 1(c) that the yield of lignin increased rapidly with the increment of concentration before $80 \%$, but when the concentration exceeds $80 \%$, the yield of lignin changes little.

3.5. Influence of Ratio of Residue 1 to Ethanediol Solution on the Yield of Lignin. The effect of the ratio of residue 1 to ethanediol solution, $1: 5,1: 6,1: 7,1: 8$, and $1: 9$, (g:mL) was, respectively, investigated. The concentration of ethanediol aqueous solution is fixed at $80 \%$, and the autoclaves were nonisothermally heated to $483 \mathrm{~K}$ and kept for a further four hours. The influence of the ratio of residue 1 to ethanediol solution on the yield of lignin is shown in Figure 1(d).

As Figure $1(\mathrm{~d})$ shows, the ratio of residue 1 to ethanediol solution has no remarkable influence on the yield of lignin. The lowest and highest yield are $14.61 \%$ and $20.20 \%$, respectively. This phenomenon may imply that the ethanediol solution is always enough for the dissolution of lignin.

The influence of pulping temperature, time, concentration of ethanediol, and the ratio of residue 1 to solution on the yield of lignin was discussed by the one-factor experiments here. However, those experiments cannot give the best combination of four factors or the key variable on the yield of lignin. So an orthogonal test design is carried out to optimize the lignin extraction; the details are shown as follows.

3.6. The Optimization of Ethanediol Pulping via the Orthogonal Experimental Design. The levels of each factor in orthogonal experimental are selected based on the one-factor 


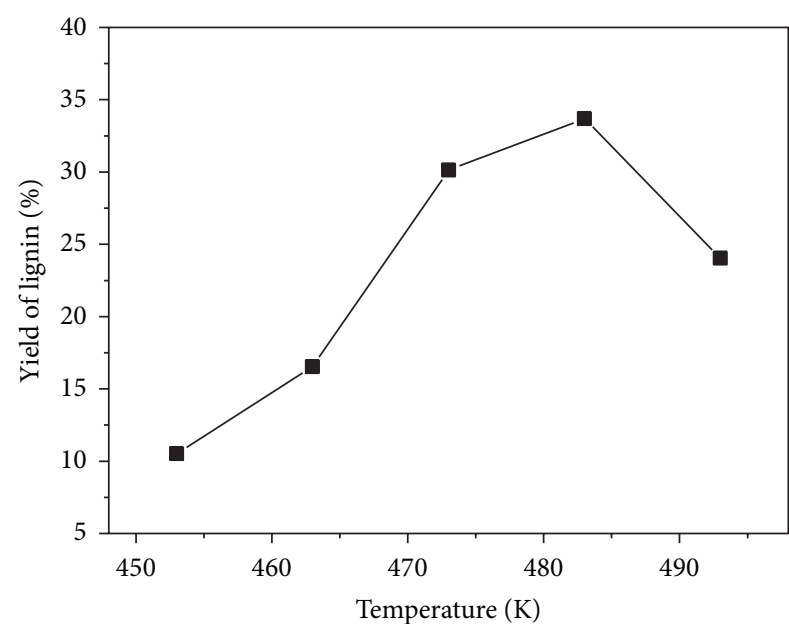

(a)

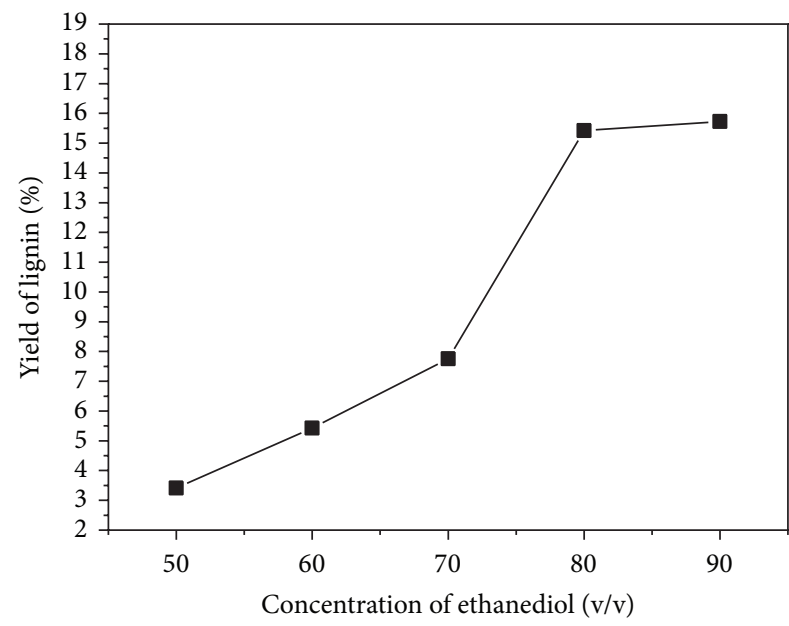

(c)

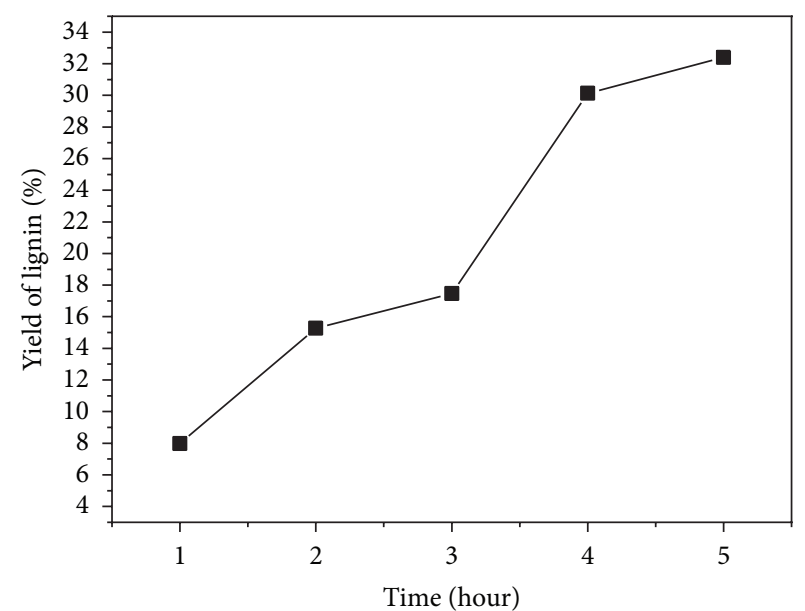

(b)

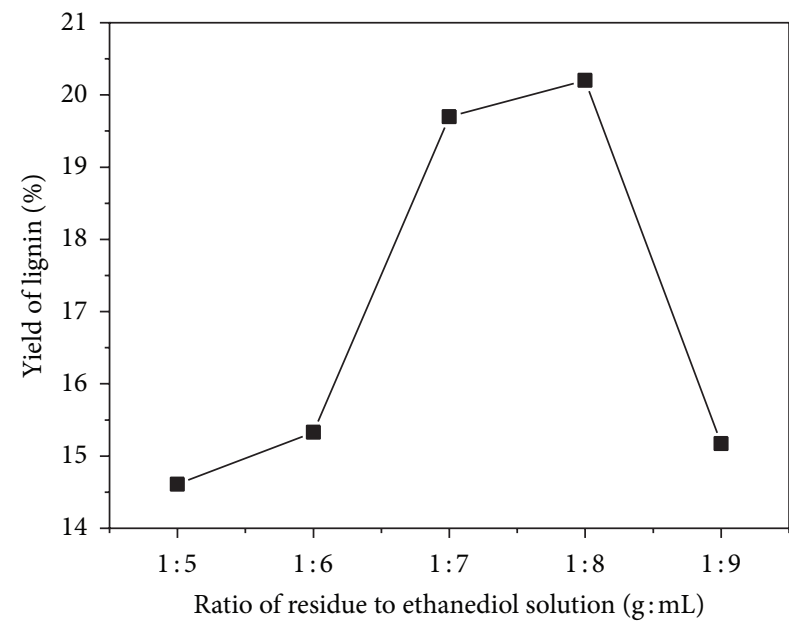

(d)

FIGURE 1: Effects of (a) temperature; (b) time; (c) concentration of ethanediol; and (d) ratio on the yields of lignin.

experimental results. For example, the levels of temperature are chosen as 473,483 , and $493 \mathrm{~K}$, for the one-factor experiments of temperature demonstrate that the optimal temperature is $483 \mathrm{~K}$, so the value around $483 \mathrm{~K}$ is chosen for the orthogonal test. Independent variables with four varying levels, A (ethanediol concentration), B (ratio of residue 1 to ethanediol), $\mathrm{C}$ (time), and D (temperature), are listed in Table 2.

All the selected factors are examined using a $\mathrm{L}_{9}(3)^{4}$ orthogonal test design. The total evaluation index is analyzed by statistical method. The analysis results performed by statistical software SPSS 13.0 are presented in Table 3 .

According to the $\mathrm{R}$ value, the factors affecting the yield (\%) of lignin are listed in a decreasing order as follows: D > $\mathrm{A}>\mathrm{C}>\mathrm{B}$. The key factor influencing the yield is temperature. The ethanediol's concentration has a remarkable influence on the yield of lignin, but the ratio of residue 1 to the ethanediol has little effect, which agrees with the results of one-factor experiments. The maximum yield $(34.47 \%)$ of the lignin is obtained when the temperature, ethanediol concentration,
TABLE 2: Factors and levels for $\mathrm{L}_{9}(3)^{4}$ orthogonal test.

\begin{tabular}{lccc}
\hline Variable & \multicolumn{3}{c}{ Levels } \\
& 1 & 2 & 3 \\
\hline $\begin{array}{l}\text { Ethanediol's concentration (v/v) } \\
\text { Ratio of residue to ethanediol } \\
(\mathrm{g}: \mathrm{mL})\end{array}$ & 70 & 80 & 90 \\
Time (hour) & $1: 4$ & $1: 6$ & $1: 8$ \\
Temperature $(\mathrm{K})$ & 4 & 5 & 6 \\
\hline
\end{tabular}

time, and ratio of residue 1 to ethanediol are $493 \mathrm{~K}, 90 \%, 4 \mathrm{~h}$, and $1: 6$, respectively.

Although the optimal condition is discussed by orthogonal test, the highest lignin's yield is only $34.47 \%$. The reason for this condition is due to the lack of semicellulose in the RH residue 1 , for the lignin matrix is broken by the acetic acid generated from the acetyl group of semicellulose [20]. 
TABLE 3: Analysis of $\mathrm{L}_{9}(3)^{4}$ orthogonal test results.

\begin{tabular}{|c|c|c|c|c|c|}
\hline Number & (A) Ethanediol concentration & (B) Ratio of residue 1 to ethanediol & (C) Time & (D) Temperature & The yield of lignin \\
\hline 1 & 1 & 1 & 1 & 1 & 10.64 \\
\hline 2 & 1 & 2 & 2 & 2 & 16.01 \\
\hline 3 & 1 & 3 & 3 & 3 & 24.01 \\
\hline 4 & 2 & 1 & 2 & 3 & 26.78 \\
\hline 5 & 2 & 2 & 3 & 1 & 18.86 \\
\hline 6 & 2 & 3 & 1 & 2 & 28.27 \\
\hline 7 & 3 & 1 & 3 & 2 & 33.60 \\
\hline 8 & 3 & 2 & 1 & 3 & 34.47 \\
\hline 9 & 3 & 3 & 2 & 1 & 18.09 \\
\hline $\mathrm{K} 1$ & 16.89 & 23.67 & 24.46 & 15.86 & \\
\hline $\mathrm{K} 2$ & 24.64 & 23.11 & 20.29 & 25.96 & \\
\hline K3 & 28.72 & 23.46 & 25.49 & 28.42 & \\
\hline$R$ & 11.83 & 0.56 & 5.20 & 12.56 & \\
\hline
\end{tabular}

Therefore, the experiments of the catalyst's influence on the yield of lignin are carried out. The sulfuric acid is chosen to provide the $\mathrm{H}^{+}$for its thermostability and less volatility.

3.7. Influence of Catalyst on the Yield of Lignin. The optimal conditions of orthogonal test are adopted, that is, $473 \mathrm{~K}$, 4 hours, $1: 6$, and $90 \%$ in the catalytic experiments. The concentrated sulfuric acid is added in the mixer of residue 1 and ethanediol solution before the pulping. The dosage of catalyst is counted by the volume percentage of ethanediol solution. Figure 2(a) shows the effects of catalyst on the yield of lignin.

It is seen from Figure 2(a) that sulfuric acid has a remarkable influence on the yield of lignin. The lignin's yield increased rapidly with the increment of catalyst's dosage. When the dosage of catalyst is $0.5 \%$, the yield of lignin reached the highest value of $58.45 \%$, which increased $70 \%$ more than the optimal result of orthogonal test. However, when the dosage of catalyst exceeded $0.5 \%$, the lignin's yield dropped due to the subsidiary reaction. The experiment showed the residue had slight carbonization at the same time.

The lignin obtained from this optimal condition is investigated by the infrared spectrometer (IR), ultraviolet spectrometer (UV), and Gel Permeation Chromatography (GPC).

The IR spectrograph and explanation are shown in Figure 2(b) and Table 4, which show the characteristic absorption peak of lignin, such as $820 \mathrm{~cm}^{-1}, 1201 \mathrm{~cm}^{-1}$, and $1275 \mathrm{~cm}^{-1}$. This result indicates the lignin structure of the sample.

The UV spectrograph is shown in Figure 2(c), which also confirms the character structure of lignin. The strongest peak near $210 \mathrm{~nm}$ is an absorption band of the conjugative bond of alkenes, indicating $n \rightarrow \pi^{*}$ electron transition and stronger undersaturation. The broad acromion that appeared around $300 \mathrm{~nm}$ is caused by the existence of the conjugated system such as aromatic ring.

GPC analysis is performed to determine $\mathrm{Mw}, \mathrm{Mn}$, and DPI of the lignin. As Figure 2(d) shows, the elution time
TABLE 4: Explanation of IR spectrograph.

\begin{tabular}{|c|c|}
\hline Position $/ \mathrm{cm}^{-1}$ & Band origin \\
\hline 3400 & $-\mathrm{OH}$ stretching \\
\hline 2943 & $\mathrm{C}-\mathrm{H}$ stretching \\
\hline 1706 & $\begin{array}{l}\mathrm{C}=\mathrm{O} \text { stretching nonconjugated } \\
\text { to the aromatic ring }\end{array}$ \\
\hline 1610 & $\begin{array}{l}\mathrm{C}=\mathrm{O} \text { stretching conjugated to the } \\
\text { aromatic ring }\end{array}$ \\
\hline 1520 & Aromatic ring vibrations \\
\hline 1462 & $\begin{array}{l}\text { Aromatic ring vibrations } \mathrm{C}-\mathrm{H} \\
\text { deformations }\end{array}$ \\
\hline 1275 & $\begin{array}{l}\text { Aromatic ring vibrations of } \\
\text { guaiacyl }\end{array}$ \\
\hline 1201 & $\mathrm{C}-\mathrm{O}$ stretching of syringy \\
\hline 1124 & Aromatic ester \\
\hline 1027 & $\mathrm{C}-\mathrm{H}$ and $\mathrm{C}-\mathrm{O}$ deformation \\
\hline 820 & Syringy $\mathrm{C}-\mathrm{H}$ vibrations \\
\hline
\end{tabular}

shifted towards higher molecular weights, and the GPC trace remained monomodal. The $\mathrm{Mw}$ and $\mathrm{Mn}$ are 1527 and 1208, respectively. The polydispersity of the polymers is 1.26.

Through the catalyzed ethanediol pulping, the optimal conditions of lignin separation from residue 1 were investigated. The highest yield of lignin reaches $58.45 \%$. The residue produced in the optimal catalyzed ethanediol pulping is named residue 2 . It has more silica and less lignin than residue 1 (as shown in Table 1), which means the decrement of lignin waste and energy consumption in the pyrolysis progress. At the same time, most of metal elements in $\mathrm{RH}$ (such as $\mathrm{K}, \mathrm{Na}$, $\mathrm{Ca}$, and $\mathrm{Al}$ ) decreased dramatically after the semicellulose hydrolysis and lignin separation (see Table 5). So residue 2 is better than residue 1 to prepare the ultrafine silica. The experiments are carried out as follows.

3.8. Preparing Amorphous Ultrafine Silica from Residues via Hydrolysis. Variables such as temperature, atmosphere, and 


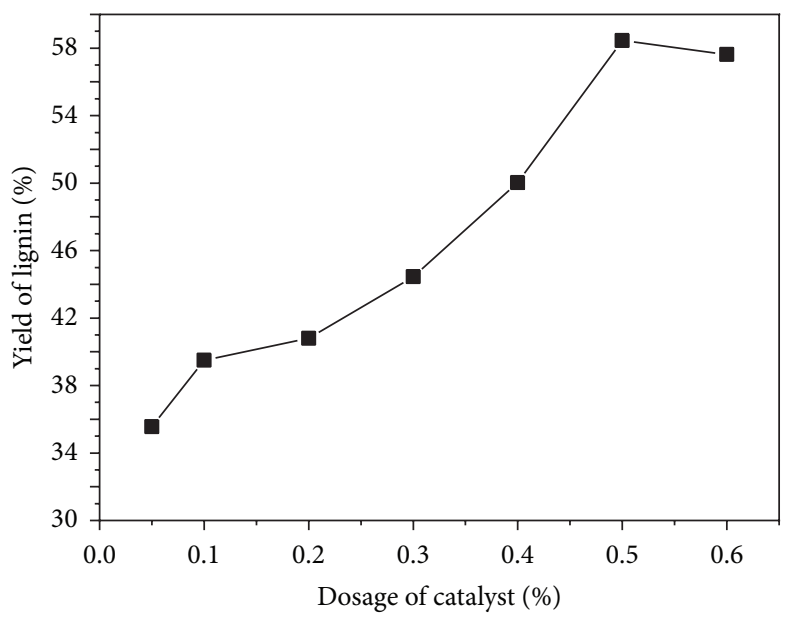

(a)

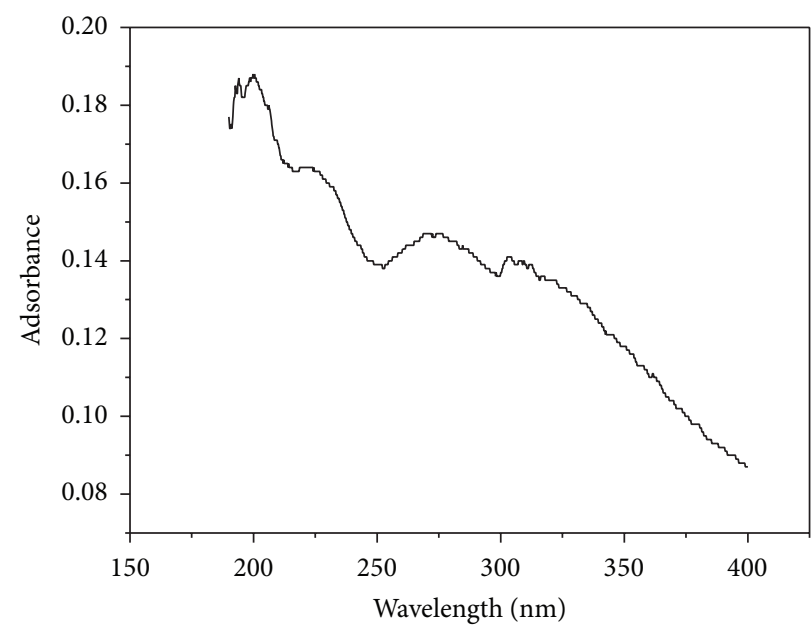

(c)

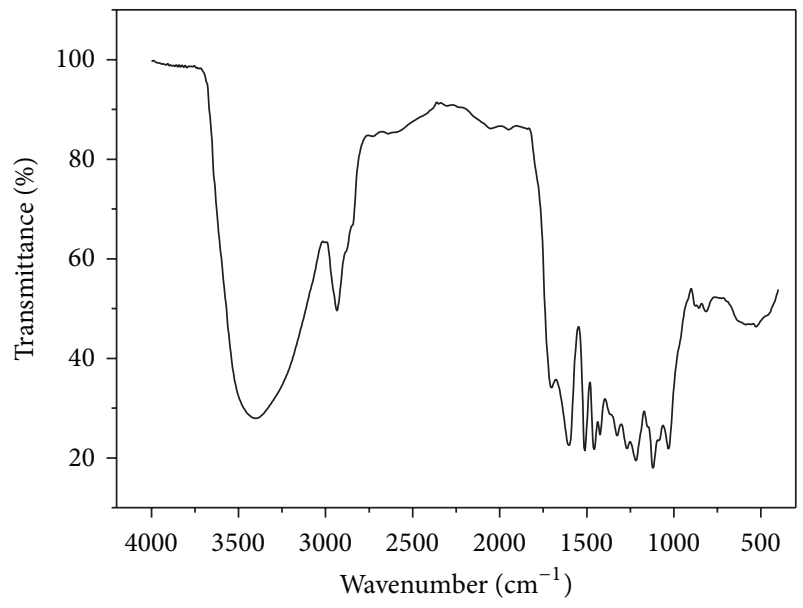

(b)

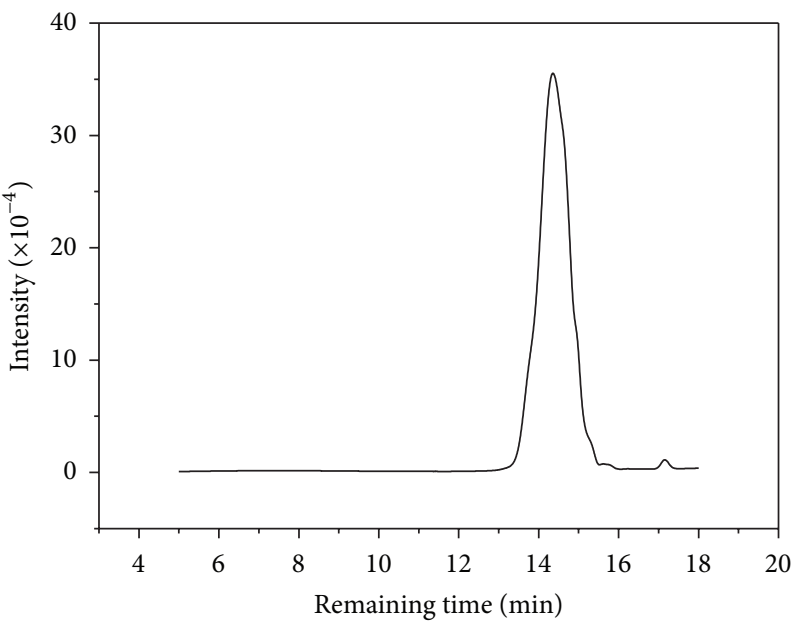

(d)

FIGURE 2: Effects of catalyst on the yield of lignin (a) and analysis of lignin: IR (b); UV (c); GPC (d).

TABLE 5: Contents of metal oxide in silica obtained from the rice husk and residue 2 under the optimum conditions of ethanediol pulping.

\begin{tabular}{lcccccccccc}
\hline Metal oxide (wt.\%) & $\mathrm{K}_{2} \mathrm{O}$ & $\mathrm{Na}_{2} \mathrm{O}$ & $\mathrm{CaO}$ & $\mathrm{MgO}$ & $\mathrm{Al}_{2} \mathrm{O}_{3}$ & $\mathrm{Fe}_{2} \mathrm{O}_{3}$ & $\mathrm{CuO}$ & $\mathrm{MnO}_{2}$ & $\mathrm{ZnO}$ & $\mathrm{Sum}$ \\
\hline From RH & 0.9808 & 0.3689 & 7.9216 & 1.7877 & 0.4530 & 0.3158 & 0.0047 & 0.1121 & 0.1145 & 12.0616 \\
From residue 2 & 0.0113 & 0.0145 & 0.0235 & 0.0011 & 0.0124 & 0.0035 & 0.0017 & 0.0000 & 0.0036 & 0.0716 \\
\hline
\end{tabular}

time have pronounced effects on the morphology and dispersity of ultrafine silica via pyrolysis of residue 2 . The details are discussed as follows.

3.9. The Temperature of Pyrolysis. Temperature has important impact on the purity and morphology of ultrafine silica. Therefore, the TG analysis was employed to investigate the pyrolysis temperature. The results of rice husk, residue 1 , and residue 2 were shown in Figure 4(a).

Figure 4(a) shows that the temperatures of constant weight for three samples increased with the increment of the ash contents, that is, $560^{\circ} \mathrm{C}$ for rice husk, $630^{\circ} \mathrm{C}$ for residue 1 , and $680^{\circ} \mathrm{C}$ for residue 2 . The reason for this difference is due to the decrease of semicellulose in residues. The thermostability of semicellulose, lignin, and cellulose is progressively increasing. Rice husk has the most abundant semicellulose; residue 1 has the most abundant lignin; residue 2 has the most abundant cellulose. Therefore, the rice husk has the lowest constant temperature for the most abundant semicellulose, and residue 2 has the highest constant temperature for the most abundant cellulose.

3.10. The Atmosphere and Time of Pyrolysis. The atmosphere and time have obvious effect on the morphology and the disparity of ultrafine silica. Experiments demonstrated that the direct hydrolysis of residue 1 in air atmosphere needs harsh conditions $\left(750^{\circ} \mathrm{C}\right.$ for 15 minutes or $600^{\circ} \mathrm{C}$ for six hours); the 


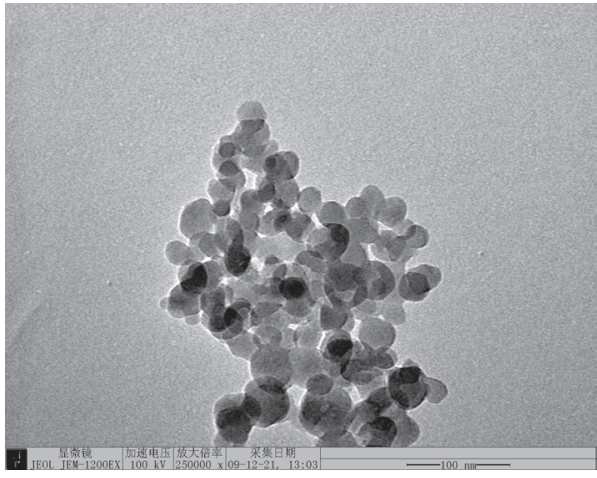

(a)

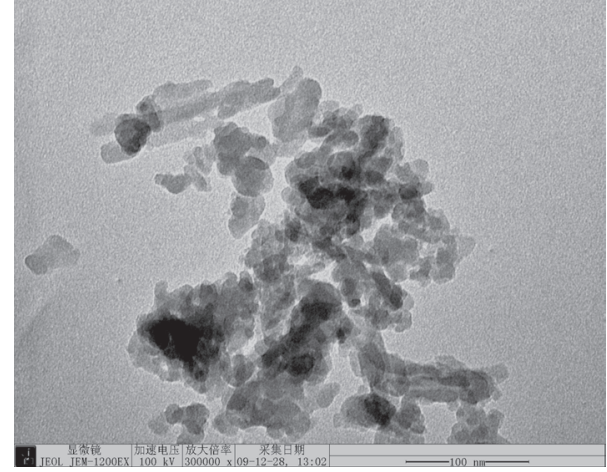

(b)

FIgURE 3: TEM of silica and carbon mixer (a) and ultrafine silica (b).
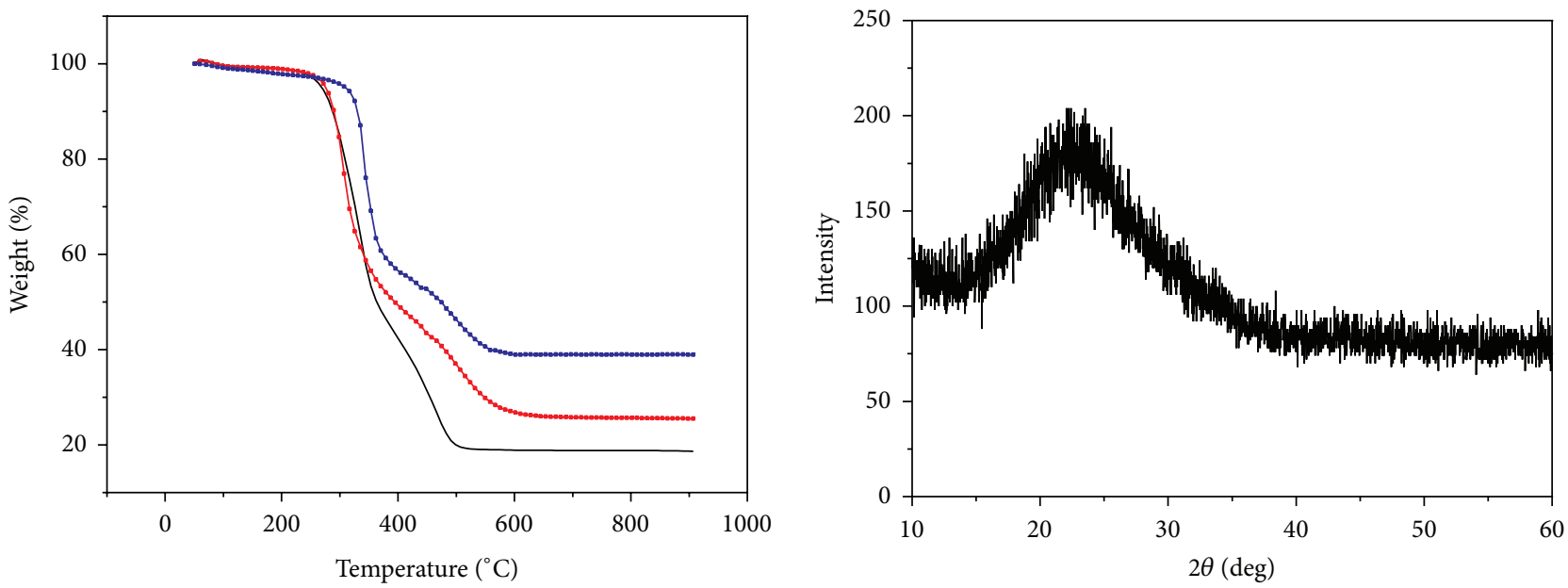

_ Rice husk

$\rightarrow$ Residue 1

$\rightarrow$ Residue 2

(a)

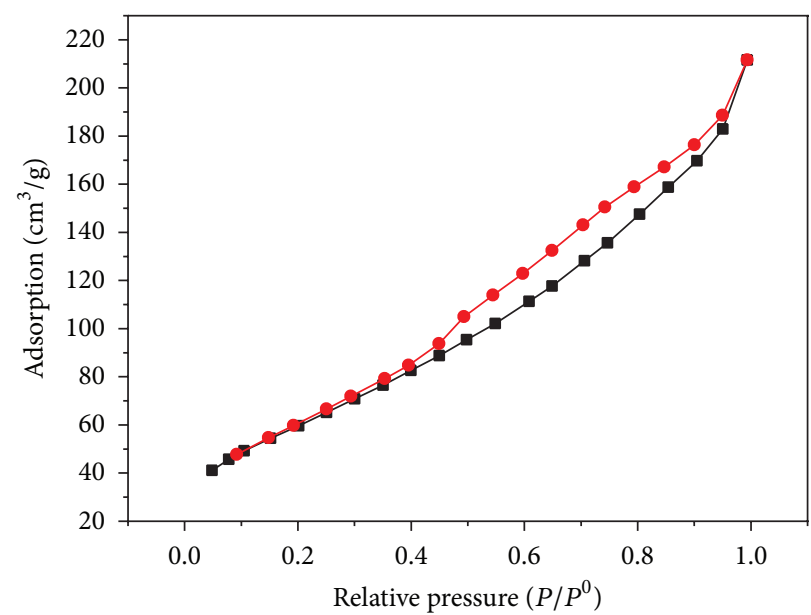

- Adsorption

$\rightarrow$ Desorption

(c)

(b)

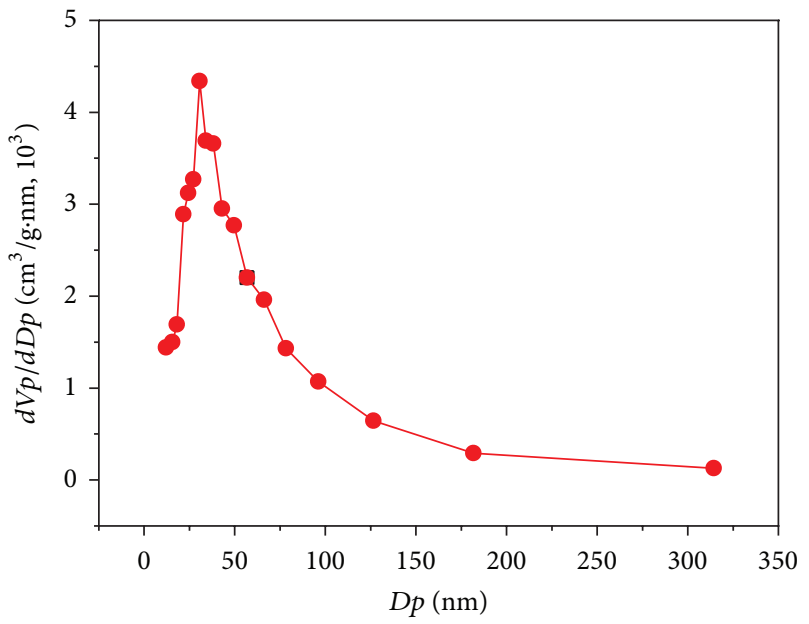

(d)

FIGURE 4: TG of rice husk and residues (a) and analysis of ultrafine silica; XRD (b); adsorption-desorption isotherms (c); differential pore size distribution (d). 
ultrafine silica had obvious agglomeration, too [18]. It is found that oxygen atmosphere can decrease the pyrolysis temperature and time comparing to the air atmosphere $\left(600^{\circ} \mathrm{C}\right.$ for 1 hour, $550^{\circ} \mathrm{C}$ for 1.5 hours), but the ultrafine silica still keeps the obvious agglomeration. The results of TG indicated that residue 2 is more difficult to pyrolyze for its high content of cellulose. Therefore, an indirect pyrolysis method is adopted to decrease the temperature of pyrolysis of residue 2 , for the sake of improving the agglomeration of silica. This method includes two steps; therefore it is called the two-step pyrolysis method in this paper.

(1) Pyrolyzing under Inert Atmosphere (The First Step). The dried residue 2 was weighted and put into a tube furnace, which was heated to $550^{\circ} \mathrm{C}$ beforehand. Residue 2 was pyrolyzed under the flowing of carbon dioxide gas for half an hour. For the inert characters of carbon dioxide, there was not the kindle of residue 2 , which avoided the remarkable increment of temperature. The organic materials such as cellulose were hydrolyzed to the carbon, which kept the silica away from agglomeration. After the first step, the mixer of carbon and silica was obtained as Figure 3(a) shows.

(2) Pyrolyzing under Oxygen Atmosphere (The Second Step). The carbon dioxide gas flow is changed to the oxygen flow under $550^{\circ} \mathrm{C}$. The black powder completely turns into white silica in 30 minutes, with the carbon being pyrolyzed to carbon dioxide.

Therefore, the temperature of pyrolyzing residue 2 is decreased to $550^{\circ} \mathrm{C}$ by the two-step pyrolysis method, which is lower than the temperature of pyrolysis in air $\left(680^{\circ} \mathrm{C}\right)$. It is helpful to improve the agglomeration of super fine silica powder.

The morphology, size, and aggregation of this silica powder are investigated by TEM. As Figure 3(b) shows, the powder is a kind of irregular aggregate with a diameter of $20 \mathrm{~nm}$.

The crystalline state of silica powder is tested by XRD, as Figure 4(b) shows. The result shows that the silica powder is amorphous state. The silica powder is porous; the BrunauerEmmett-Teller (BET) method is used to analyze the porosity and specific surface area of the sample. Figure 4(c) shows the nitrogen adsorption-desorption isotherms of silica powder at $77 \mathrm{~K}$. The lower portion of the loop is traced out on adsorption, and the upper portion on desorption. It is seen that the hysteresis loop resembles type IV of Brunaur's classification. At low values of $\mathrm{P} / \mathrm{P}^{0}$, the isotherm is similar to type II, but then adsorption increases markedly at $\mathrm{P} / \mathrm{P}^{0}$ above 0.5 , where pore condensation takes place.

Figure 4(d) shows a plot of the increment of pore volume per increment in pore size, versus pore size. This result is determined by the Barrett-Joyner-Halenda $(\mathrm{BJH})$ method (based on the desorption branch). A single peak is observed in the silica samples having maxima at $30.6 \mathrm{~nm}$. The pores may result from the residual spaces between the fine particles existing or formed in the silica preparation. The BET result tells the specific surface area of this silica is $225.20 \mathrm{~m}^{2} / \mathrm{g}$. The reason for porosity is that the organic matter has been broken up during the thermal decomposition of rice husk, thus leaving a highly porous structure.

The purity for silica obtained from the rice husk and residue 2 under the optimum conditions of ethanediol pulping is investigated by ICP. The results are shown in Table 5. It is seen that the metal oxides in $\mathrm{RH}$ are removed efficiently after semicellulose hydrolysis and ethanediol pulping. The purity of silica is $99.92 \%$, which is higher than the silica obtained from residue 1 via pyrolyzing in air atmosphere [18], with better dispersity and lower temperature of residue pyrolyzing.

\section{Conclusions}

Through sulfuric acid hydrolysis, ethanediol solution pulping, and the two-step pyrolysis, the high purity D-xylose, organosolv lignin, and ultrafine silica are prepared consecutively from rice husk, so the semicellulose, lignin, and silica in rice husk are comprehensively utilized. The optimal conditions for extracting lignin from residue 1 are $493 \mathrm{~K}, 90 \%(\mathrm{v} / \mathrm{v})$, $4 \mathrm{~h}$, and $1: 6(\mathrm{~g}: \mathrm{mL})$, with the maximum yield of $34.47 \%$. When the dosage of catalyst (concentrated sulfuric acid) is $0.5 \%$, the yield of lignin reached the highest $58.45 \%$. Amorphous ultrafine silica is prepared from residual 2 via twostep pyrolysis method with the diameter around $20 \mathrm{~nm}$, the specific surface of $225.20 \mathrm{~m}^{2} / \mathrm{g}$, and the purity of $99.92 \%$ (wt.\%).

\section{Conflict of Interests}

The authors declare that there is no conflict of interests regarding the publication of this paper.

\section{Acknowledgment}

The authors gratefully acknowledge the Department of Science and Technology of Jilin Province (no. 20130206076GX, no. 20130206056GX) for their financial supports.

\section{References}

[1] Y. Qu, Y. Tian, B. Zou et al., "A novel mesoporous lignin/silica hybrid from rice husk produced by a sol-gel method," Bioresource Technology, vol. 101, no. 21, pp. 8402-8405, 2010.

[2] G. Görhan and O. Şimsek, "Porous clay bricks manufactured with rice husks," Construction and Building Materials, vol. 40, pp. 390-396, 2013.

[3] Y. P. Guo, S. F. Yang, J. Z. Zhao, Z. C. Wang, and M. Y. Zhao, "Preparation of active carbon with high specific surface area from rice husks," Chemical Journal of Chinese Universities, vol. 21, no. 3, pp. 335-338, 2000.

[4] O. C. Okeh, C. O. Onwosi, and F. J. C. Odibo, "Biogas production from rice husks generated from various rice mills in Ebonyi State, Nigeria," Renewable Energy, vol. 62, pp. 204-208, 2014.

[5] F. Adam, K. Kandasamy, and S. Balakrishnan, "Iron incorporated heterogeneous catalyst from rice husk ash," Journal of Colloid and Interface Science, vol. 304, no. 1, pp. 137-143, 2006.

[6] T. Watanabe, M. Shida, T. Murayama et al., "Xyloglucan in cell walls of rice hull," Carbohydrate Research, vol. 129, pp. 229-242, 1984. 
[7] Y. Yu, X. Lou, and H. Wu, "Some recent advances in hydrolysis of biomass in hot-compressed water and its comparisons with other hydrolysis methods," Energy and Fuels, vol. 22, no. 1, pp. 46-60, 2008.

[8] W. Mu, H. Ben, A. Ragauskas, and Y. Deng, "Lignin pyrolysis components and upgrading-technology review," Bioenergy Research, vol. 6, no. 4, pp. 1183-1204, 2013.

[9] V. Ganvir and K. Das, "Removal of fluoride from drinking water using aluminum hydroxide coated rice husk ash," Journal of Hazardous Materials, vol. 185, no. 2-3, pp. 1287-1294, 2011.

[10] W. van Beinum, S. Beulke, and C. D. Brown, "Pesticide sorption and desorption by lignin described by an intraparticle diffusion model," Environmental Science \& Technology, vol. 40, no. 2, pp. 494-500, 2006.

[11] Suhas, P. J. M. Carrott, and M. M. L. Ribeiro Carrott, "Ligninfrom natural adsorbent to activated carbon: a review," Bioresource Technology, vol. 98, no. 12, pp. 2301-2312, 2007.

[12] G. J. Cui, W. B. Xia, G. J. Chen, M. Wei, and J. Huang, "Enhanced mechanical performances of waterborne polyurethane loaded with lignosulfonate and its supramolecular complexes," Journal of Applied Polymer Science, vol. 106, no. 6, pp. 4257-4263, 2007.

[13] M. A. Gilarranz, A. Santos, J. García, M. Oliet, and F. Rodríguez, "Kraft pulping of Eucalyptus globulus: kinetics of residual delignification," Industrial and Engineering Chemistry Research, vol. 41, no. 8, pp. 1955-1959, 2002.

[14] S. Gu, J. Zhou, Z. Luo, Q. Wang, and M. Ni, "A detailed study of the effects of pyrolysis temperature and feedstock particle size on the preparation of nanosilica from rice husk," Industrial Crops and Products, vol. 50, pp. 540-549, 2013.

[15] N. S. C. Zulkifli, I. Ab Rahman, D. Mohamad, and A. Husein, "A green sol-gel route for the synthesis of structurally controlled silica particles from rice husk for dental composite filler," Ceramics International, vol. 39, no. 4, pp. 4559-4567, 2013.

[16] K. P. Dey, S. Ghosh, and M. K. Naskar, "Organic template-free synthesis of ZSM-5 zeolite particles using rice husk ash as silica source," Ceramics International, vol. 39, no. 2, pp. 2153-2157, 2013.

[17] V. B. Carmona, R. M. Oliveira, W. T. L. Silva, L. H. C. Mattoso, and J. M. Marconcini, "Nanosilica from rice husk: extraction and characterization," Industrial Crops and Products, vol. 43, no. 1, pp. 291-296, 2013.

[18] H. Zhang, X. Zhao, X. Ding et al., "A study on the consecutive preparation of $\mathrm{d}$-xylose and pure superfine silica from rice husk," Bioresource Technology, vol. 101, no. 4, pp. 1263-1267, 2010.

[19] T. Yokoyama and Y. Matsumoto, "Revisiting the mechanism of $\beta$-O-4 bond cleavage during acidolysis of lignin. Part 2: detailed reaction mechanism of a non-phenolic $\mathrm{C}_{6}-\mathrm{C}_{2}$ type model compound," Journal of Wood Chemistry and Technology, vol. 30, no. 3, pp. 269-282, 2010.

[20] T. Kishimoto, A. Ueki, H. Takamori, Y. Uraki, and M. Ubukata, "Delignification mechanism during high-boiling solvent pulping. Part 6: changes in lignin structure analyzed bylH-13C correlation 2-D NMR spectroscopy," Holzforschung, vol. 58, no. 4, pp. 355-362, 2004. 

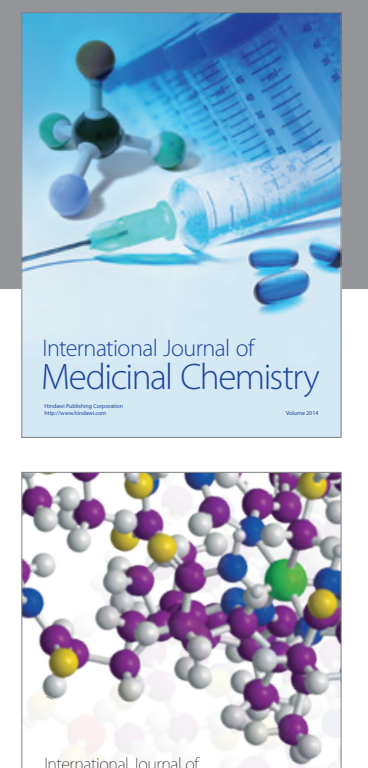

\section{Carbohydrate} Chemistry

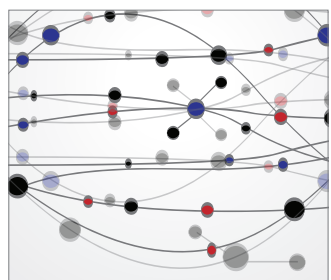

The Scientific World Journal
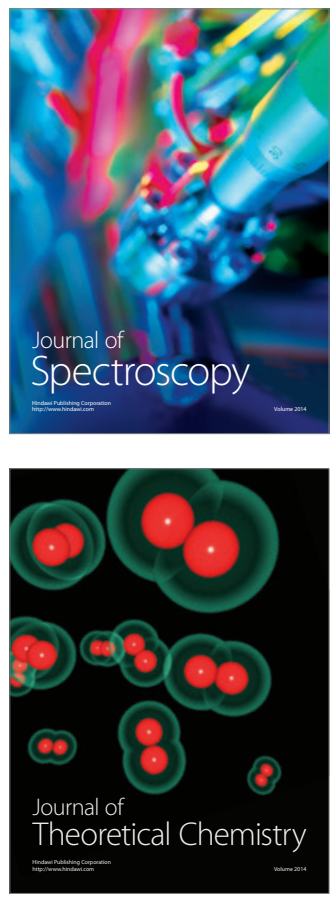
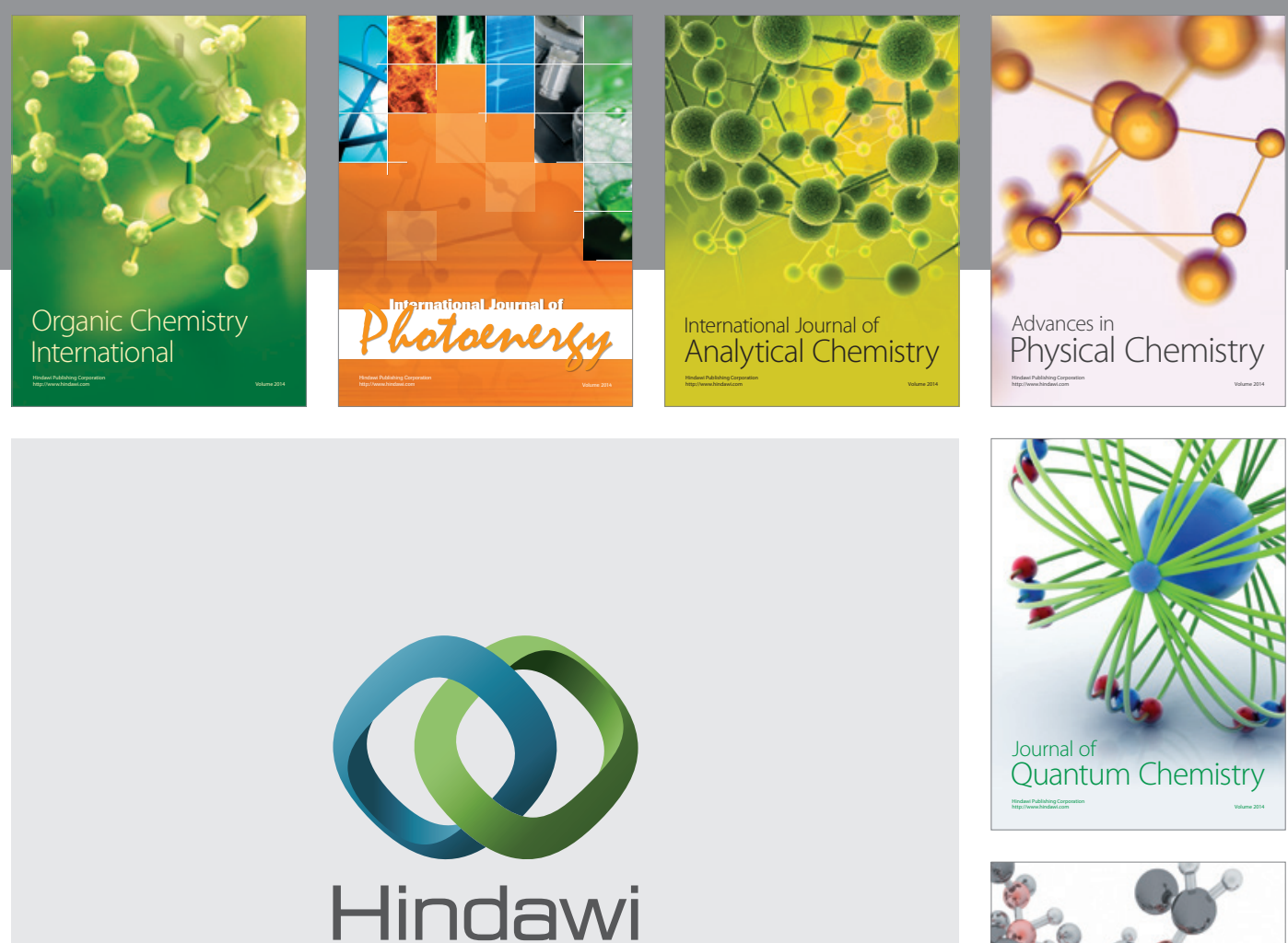

Submit your manuscripts at

http://www.hindawi.com

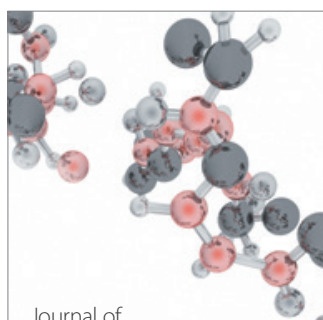

Analytical Methods

in Chemistry

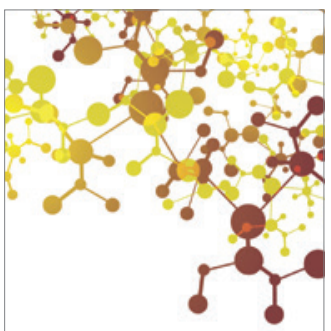

Journal of

Applied Chemistry

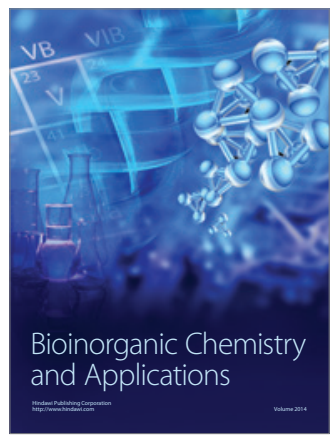

Inorganic Chemistry
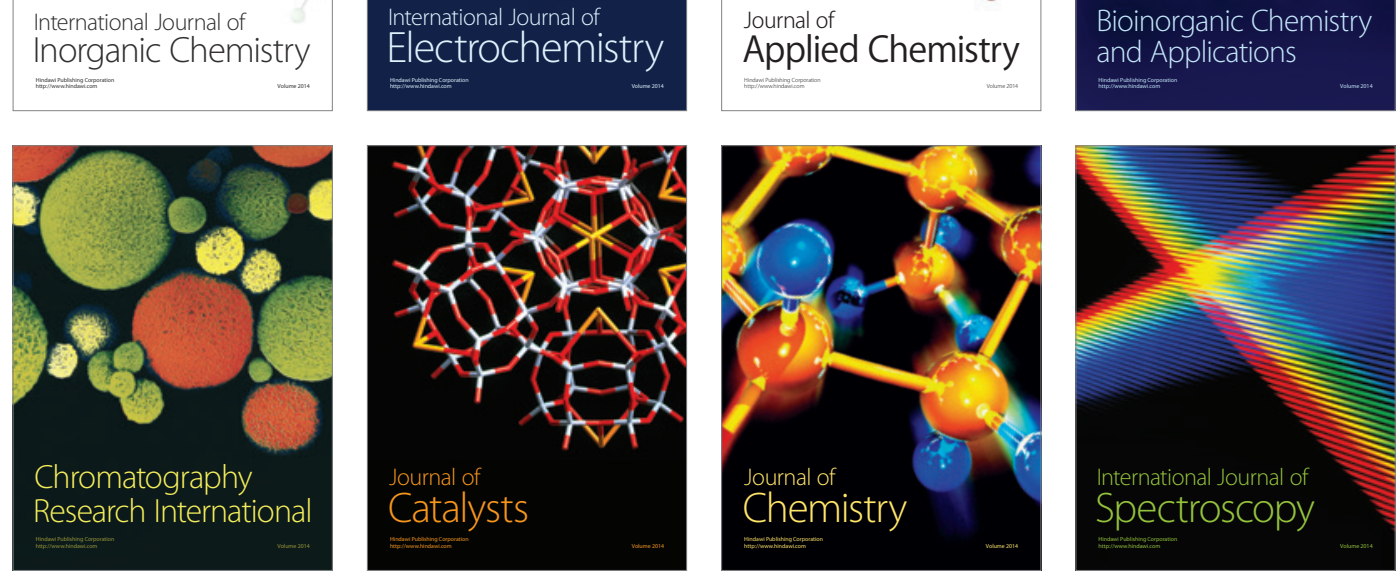